Portland State University

PDXScholar

Electrical and Computer Engineering Faculty

Publications and Presentations

10-1-1973

\title{
Gaussian Light Beams in Inhomogeneous Media
}

Lee W. Casperson

Portland State University

Follow this and additional works at: https://pdxscholar.library.pdx.edu/ece_fac

Part of the Electrical and Computer Engineering Commons

Let us know how access to this document benefits you.

\section{Citation Details}

Lee W. Casperson, "Gaussian Light Beams in Inhomogeneous Media," Appl. Opt. 12, 2434-2441 (1973).

This Article is brought to you for free and open access. It has been accepted for inclusion in Electrical and Computer Engineering Faculty Publications and Presentations by an authorized administrator of PDXScholar. Please contact us if we can make this document more accessible: pdxscholar@pdx.edu. 


\title{
Gaussian Light Beams in Inhomogeneous Media
}

\author{
Lee W. Casperson
}

\begin{abstract}
Vector wave solutions are obtained for the propagation of beams of light in media having slow spatial variations of the gain, loss, or index of refraction. The formalism developed here is applicable to a wide range of problems, and an example considered in detail is the propagation of off-axis beams in lenslike laser materials and optical waveguides. A procedure is also described for the diagnosis of localized dielectric inhomogeneities such as plasmas by means of Gaussian laser beams.
\end{abstract}

\section{Introduction}

The output beam modes of most conventional lasers can be simply described in terms of HermiteGaussian $^{1}$ or Laguerre-Gaussian ${ }^{2}$ functions. Therefore, for applications involving the propagation of laser beams it is important to understand what effects various media and optical systems will have on such Gaussian beams. The purpose of this work is to examine the propagation of Gaussian beams in media having slow spatial variations of the gain and index of refraction. It is assumed here that the variations are sufficiently slow in the vicinity of the beam that the complex propagation constant can be expanded keeping only the constant, linear, and quadratic terms. If this condition is satisfied, a propagating beam will remain Guassian even though its spot size, phase front curvature, amplitude, phase, and direction of propagation are significantly altered by the inhomogeneous medium.

Slow variations of the propagation constant occur very commonly in practice and they may be either intentional or unavoidable. For example, in gas lasers there may be spatial profiles due to the free electrons ${ }^{3}$ or the gain ${ }^{4,5}$ and dispersion ${ }^{6}$ of the laser transition or a neighboring transition. ${ }^{7}$ In $\mathrm{cw}$ solid lasers index profiles due to thermal effects are common. ${ }^{8}$ Lenslike media have also been proposed as a means for propagating information-bearing light beams over long distances, ${ }^{9-12}$ and the recently developed SELFOC lenslike optical waveguides have been designed for this purpose. ${ }^{13}$ Such profiles may also occur naturally in the atmosphere and affect the long-distance propagation of beams. Most treatments related to this subject are specialized in nature and begin with either the ray equation or the scalar wave equation. Here we develop a general wave equation formalism and obtain the components

The author is with the School of Engineering and Applied Science, University of California, Los Angeles, California 90024.

Received 20 February 1973. of the vector waves. The validity of the ray equation in treating beam propagation is also considered.

In Sec. II the wave equation is reduced to a much simpler set of ordinary differential equations governing all the parameters of a Gaussian beam in an inhomogeneous material, and in the following sections solutions of these equations are obtained for several situations of practical interest. The problem of off-axis beams in complex lenslike media is discussed in Sec. III and it is shown that gain or loss profiles strongly affect the stability of long-distance beam propagation. Section IV contains a detailed solution for steady-state higher order beam propagation. In Sec. V a procedure is developed for diagnosing localized inhomogeneous refracting and absorbing media such as plasmas by means of Gaussian laser beams.

\section{Derivation of Beam Equations}

A Gaussian beam can be completely characterized by a small number of parameters that describe the size, location, and phase properties of the beam. In this section the basic equations governing these parameters are derived using conventional beam notation. The starting point for this derivation is Maxwell's equations, which for harmonically varying fields in an isotropic medium may be written

$$
\bar{\nabla} \times \bar{E}=-i \omega \mu \bar{H}, \quad \bar{\nabla} \times \bar{H}=i \omega \epsilon \bar{E},
$$

where the permittivity $\epsilon$ has an imaginary part to account for conductivity and the out-of-phase component of the polarization. These equations may be combined to yield the wave equation for the electric field

$$
\bar{\nabla} \times \bar{\nabla} \times \bar{E}-\omega^{2} \mu \epsilon \bar{E}=(\bar{\nabla} \mu / \mu) \times \bar{\nabla} \times \bar{E} .
$$

For the Cartesian field components Eq. (2) becomes

$$
\nabla^{2} \bar{E}+\omega^{2} \mu \epsilon \bar{E}=-\bar{\nabla}[(\bar{\nabla} \epsilon / \epsilon) \cdot \bar{E}]-(\bar{\nabla} \mu / \mu) \times \bar{\nabla} \times \bar{E},
$$


where Gauss' law for a neutral medium $\bar{\nabla} \cdot \epsilon \overline{\bar{E}}=0$ has been used. Equation (3) is really three coupled scalar equations governing the components of the electric field.

For nearly plane waves in a weakly inhomogeneous medium the longitudinal electric field component is much smaller than the transverse components. From Eq. (3) it follows that if the changes in $\epsilon$ and $\mu$ per wavelength are small, for the dominant transverse components the right-hand side of the equation is negligible. The simplified wave equation is

$$
\nabla^{2} \bar{E}+k^{2} \bar{E}=0,
$$

where the wavenumber $k=\omega(\mu \epsilon)^{1 / 2}$ has been introduced. An identical equation is also obtained for the transverse components of the magnetic field. It is not generally true, as is sometimes implied, that Eq. (4) holds also for the weak longitudinal fields in this approximation (small change in $\epsilon$ and $\mu$ per wavelength). ${ }^{14}$ However, the longitudinal fields can be determined directly from the transverse components by means of Eq. (1) and an example is considered in Sec. IV.

We now assume specifically that the beam is polarized in the $x$ direction and is propagating more or less parallel to the $z$ axis, so that solutions of Eq. (4) will describe the $x$ component of the electric field. If $k^{2}$ has at most a quadratic $x$ and $y$ dependence in the vicinity of the beam, one can write

$$
\begin{aligned}
& k^{2}(x, y, z)=k_{0}(z)\left[k_{0}(z)-k_{1 x}(z) x-k_{1 y}(z) y\right. \\
&-\left.k_{2 x}(z) x^{2}-2 k_{x y}(z) x y-k_{2 y}(z) y^{2}\right] .
\end{aligned}
$$

This is the most general second-degree profile possible and includes most of the situations encountered in practice. The wavenumber $k$ is complex to include profiles of the gain or loss. For a wave propagating in the $z$ direction a useful substitution is

$$
E_{x}=G(x, y, z) \exp \left[-i \int k_{0}(z) d z\right] .
$$

Then Eq. (4) reduces to

$$
\begin{aligned}
& \left(\partial^{2} G / \partial x^{2}\right)+\left(\partial^{2} G / \partial y^{2}\right)-2 i k_{0}(\partial G / \partial z)-i\left(d k_{0} / d z\right) G \\
& -k_{0}\left(k_{1 x} x+k_{1 y} y+k_{2 x} x^{2}+2 k_{x y} x y+k_{2 y} y^{2}\right) G=0,
\end{aligned}
$$

where $G$ is assumed to vary so slowly with $z$ that its second derivative can be neglected.

The general astigmatic form for a Gaussian beam can be written

$$
\begin{aligned}
G(x, y, z) & =\exp -i\left\{\left[Q_{x}(z) x^{2} / 2\right]+Q_{x y}(z) x y\right. \\
+ & {\left.\left[Q_{y}(z) y^{2} / 2\right]+S_{x}(z) x+S_{y}(z) y+P(z)\right\} . }
\end{aligned}
$$

The size of the beam and the curvature of the phase fronts are governed by the complex beam parameters $Q_{x}, Q_{x y}$, and $Q_{y}$. It will be shown that the location of the beam depends on the complex displacement parameters $S_{x}$ and $S_{y}$. The phase and amplitude of the beam are governed by the complex phase parameter $P$. Other beam configurations can be expanded in terms of these fundamental astigmatic Gaussian beam modes. If Eq. (8) is substituted into Eq. (7), one finds by equating equal powers of $x, y$, and $x y$ that the beam parameters are governed by the following equations:

$$
\begin{gathered}
Q_{x}{ }^{2}+Q_{x y}{ }^{2}+k_{0}\left(d Q_{x} / d z\right)+k_{0} k_{2 x}=0 \\
Q_{y}{ }^{2}+Q_{x y}{ }^{2}+k_{0}\left(d Q_{y} / d z\right)+k_{0} k_{2 y}=0, \\
\left(Q_{x}+Q_{y}\right) Q_{x y}+k_{0}\left(d Q_{x y} / d z\right)+k_{0} k_{x y}=0 \\
Q_{x} S_{x}+Q_{x y} S_{y}+k_{0}\left(d S_{x} / d z\right)+\left(k_{0} k_{1 x}\right) / 2=0 \\
Q_{y} S_{y}+Q_{x y} S_{x}+k_{0}\left(d S_{y} / d z\right)+\left(k_{0} k_{1 y}\right) / 2=0 \\
\left(d P^{i} / d z\right)=-i\left[\left(Q_{x}+Q_{y}\right) / 2 k_{0}\right] \\
-\left[\left(S_{x}{ }^{2}+S_{y}{ }^{2}\right) / 2 k_{0}\right]-\left(i / 2 k_{0}\right)\left(d k_{0} / d z\right) .
\end{gathered}
$$

Thus the wave equation is reduced to a set of ordinary differential equations.

The procedure for determining the propagation of a Gaussian beam in a slowly varying inhomogeneous medium involves expanding $k$ in the vicinity of the beam to second order in a Taylor series to obtain the appropriate coefficients $k_{0}(z), k_{1 x}(z), k_{1 y}(z), k_{2 x}(z)$, $k_{2 y}(z)$, and $k_{x y}(z)$ while simultaneously solving Eqs. (9)-(14). This procedure is difficult in general, but numerical solutions are always possible. However, in many situations of practical interest it is valid to assume that the term $k_{x y}(z)$ in the propagation constant is equal to zero. This is simply the requirement that the symmetry axes of the quadratic approximation to the medium remain parallel to the $x$ and $y$ axes of the coordinate system. Furthermore, if the symmetry axes of the astigmatic beam are also initially parallel to the $x$ and $y$ axes $\left[Q_{x y}(0)=0\right]$, $Q_{x y}(z)=0$ according to Eq. (11). Thus Eqs. (9)(14) reduce to the much simpler set

$$
\begin{gathered}
Q_{x}{ }^{2}+k_{0}\left(d Q_{x} / \dot{d} z\right)+k_{0} k_{2 x}=0, \\
Q_{y}{ }^{2}+k_{0}\left(d Q_{y} / d z\right)+k_{0} k_{2 y}=0, \\
Q_{x} S_{x}+k_{0}\left(d S_{x} / d z\right)+\left(k_{0} k_{1 x}\right) / 2=0, \\
Q_{y} S_{y}+k_{0}\left(d S_{y} / d z\right)+\left(k_{0} k_{1 y}\right) / 2=0, \\
(d P / d z)=-i\left[\left(Q_{x}+Q_{y}\right) /\left(2 k_{0}\right)\right] \\
-\left[\left(S_{x}{ }^{2}+S_{y}{ }^{2}\right) /\left(2 k_{0}\right)\right]-\left(i / 2 k_{0}\right)\left(d k_{0} / d z\right),
\end{gathered}
$$

and the Gaussian beam has the form

$$
\begin{aligned}
& G(x, y, z)=\exp \\
& \quad-i\left(Q_{x} x^{2} / 2+Q_{y} y^{2} / 2+S_{x} x+S_{y} y+P\right) .
\end{aligned}
$$

The complex parameters $Q_{x}$ and $Q_{y}$ describe the transverse properties of the beam. It is conventional to introduce the complex beam radius $q=k_{0} / Q$, which has the form

$$
1 / q=Q / k_{0}=(1 / R)-i\left[\lambda_{m} /\left(\pi w^{2}\right)\right],
$$

where $R$ is the radius of curvature of the spherical phase fronts regarded as positive when the center of curvature is behind the phase front, $w$ is the 1/e spot size, and $\lambda_{m}$ is the wavelength in the medium. If the beam parameters are separated into their real and imaginary parts according to the form $Q_{x}=Q_{x r}$ $+i Q_{x i}, S_{x}=S_{x r}+i S_{x i}, P=P_{r}+i P_{i}$, etc., Eq. (20) can be factored as 


$$
\begin{aligned}
G=\exp \left\{-i\left[\frac{Q_{x r}}{2}\left(x-d_{x p}\right)^{2}+\frac{Q_{y r}}{2}\left(y-d_{y p}\right)^{2}\right.\right. \\
\left.-\frac{Q_{x r} d_{x p}^{2}}{2}-\frac{Q_{y r} d_{y p}^{2}}{2}+P_{r}\right] \\
+\left[\frac{Q_{x i}}{2}\left(x-d_{x a}\right)^{2}+\frac{Q_{y i}}{2}\left(y-d_{y a}\right)^{2}\right. \\
\left.\left.-\frac{Q_{x i} d_{x a}^{2}}{2}-\frac{Q_{y i} d_{y a}^{2}}{2}+P_{i}\right]\right\}
\end{aligned}
$$

where $d_{x a}=-S_{x i} / Q_{x i}$ is the displacement in the $x$ direction of the center of the amplitude distribution and $d_{x p}=-S_{x r} / Q_{x r}$ is the displacement in the $x$ direction of the center of the phase fronts. The location of the beam with respect to the $z$ axis is thus determined by the real numbers $d_{x a}$ and $d_{y a}$. Analytic solutions of the beam equations and their interpretation are included in the following sections.

\section{Solutions of Beam Equations}

We have shown that the propagation of a Gaussian beam in a slowly varying inhomogeneous medium is determined entirely by the evolution of the complex parameters $Q, S$, and $P$. In this section solutions of Eqs. (15)-(19), which govern these parameters, are discussed for several situations of practical interest. It is assumed here that $k_{0}, k_{1 x}, k_{1 y}, k_{2 x}$, and $k_{2 y}$ are known constants. In Sec. $\mathrm{V}$ an example is considered in which these parameters are functions of $z$.

Equations (15) and (16) are Ricatti equations and the solutions for $Q_{x}$ can be written in the form ${ }^{5}$

$$
\begin{aligned}
& \frac{Q_{x}(z)}{k_{0}}= \\
& \frac{-\left(k_{2 x} / k_{0}\right)^{1 / 2} \sin \left[\left(k_{2 x} / k_{0}\right)^{1 / 2} z\right]+\left[Q_{x}(0) / k_{0}\right] \cos \left[\left(k_{2 x} / k_{0}\right)^{1 / 2} z\right]}{\cos \left[\left(k_{2 . x} / k_{0}\right)^{1 / 2} z\right]+\left[Q_{x}(0) / k_{0}\right]\left(k_{0} / k_{2 x}\right)^{1 / 2} \sin \left[\left(k_{2 x} / k_{0}\right)^{1 / 2} z\right]}
\end{aligned}
$$

with a similar result for $Q_{y}(z)$. For a uniform medium $\left(k_{2 x}=0\right) \mathrm{Eq}$. (23) simplifies to $q_{x}(z)=q_{x}(0)+$ $z$. The solution of Eqs. (17)-(19) is simplified considerably by using Eqs. (15) and (16) and introducing the new parameters $S_{x}{ }^{\prime}=S_{x}-k_{1 x} Q_{x} /\left(2 k_{2 x}\right)$, $S_{y}^{\prime}=S_{y}-k_{1 y} Q_{y} /\left(2 k_{2 y}\right)$, and $P^{\prime}=P-k_{1 x} S_{x} /$ $\left(2 k_{2 x}\right)-k_{1 y} S_{y} /\left(2 k_{2 y}\right)+k_{1 x^{2}} Q_{x} /\left(8 k_{2 x^{2}}\right)+k_{1 y^{2}} Q_{y} /$

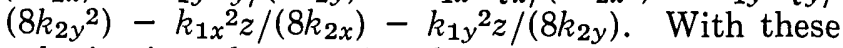
substitutions the equations become

$$
\begin{gathered}
\left(d S_{x}{ }^{\prime} / d z\right)+\left(Q_{x} S_{x}{ }^{\prime} / k_{0}\right)=0 \\
\left(d S_{y}{ }^{\prime} / d z\right)+\left(Q_{y} S_{y}{ }^{\prime} / k_{0}\right)=0 \\
\left(d P^{\prime} / d z\right)=-i\left[\left(Q_{x}+Q_{y}\right) /\left(2 k_{0}\right)\right] \\
-\left[\left({S_{x}}^{\prime 2}+S_{y}{ }^{2}\right) /\left(2 k_{0}\right)\right] .
\end{gathered}
$$

Using Eqs. (23) and (24), the solution for $S_{x}{ }^{\prime}$ is

$$
\begin{aligned}
& S_{x}{ }^{\prime}(z)=S_{x}{ }^{\prime}(0) / \\
& \quad\left\{\cos \left[\left(k_{2 x} / k_{0}\right)^{1 / 2} z\right]+\left[Q_{x}(0) / k_{0}\right]\left(k_{0} / k_{2 x}\right)^{1 / 2} \sin \left[\left(k_{2 x} / k_{0}\right)^{1 / 2} z\right]\right\}
\end{aligned}
$$

with a similar result for $S_{y^{\prime}}(z)$. Using Eqs. (23), (26), and (27), $P^{\prime}$ is found to be

$$
\begin{gathered}
P^{\prime}(z)-P^{\prime}(0)=-(i / 2) \ln \left\{\cos \left[\left(k_{2 x} / k_{0}\right)^{1 / 2} z\right]\right. \\
\left.+\left[Q_{x}(0) / k_{0}\right]\left(k_{0} / k_{2 x}\right)^{1 / 2} \sin \left[\left(k_{2 x} / k_{0}\right)^{1 / 2} z\right]\right\} \\
-(i / 2) \ln \left\{\cos \left[\left(k_{2 y} / k_{0}\right)^{1 / 2} z\right]\right. \\
\left.+\left[Q_{y}(0) / k_{0}\right]\left(k_{0} / k_{2 y}\right)^{1 / 2} \sin \left[\left(k_{2 y} / k_{0}\right)^{1 / 2} z\right]\right\} \\
-\frac{\left[S_{x}{ }^{\prime}(0)\right]^{2}}{2 k_{0}} \\
\times \frac{\left(k_{0} / k_{2 x}\right)^{1 / 2} \sin \left[\left(k_{2 x} / k_{0}\right)^{1 / 2} z\right]}{\cos \left[\left(k_{2 x} / k_{0}\right)^{1 / 2} z\right]+\left[Q_{x}(0) / k_{0}\right]\left(k_{0} / k_{2 x}\right)^{1 / 2} \sin \left[\left(k_{2 x} / k_{0}\right)^{1 / 2} z\right]} \\
-\frac{\left[S_{y}{ }^{\prime}(0)\right]^{2}}{2 k_{0}} \\
\times \frac{\left(k_{0} / k_{2 y}\right)^{1 / 2} \sin \left[\left(k_{2 y} / k_{0}\right)^{1 / 2} z\right]}{\cos \left[\left(k_{2 y} / k_{0}\right)^{1 / 2} z\right]+\left[Q_{y}(0) / k_{0}\right]\left(k_{0} / k_{2 y}\right)^{1 / 2} \sin \left[\left(k_{2 y} / k_{0}\right)^{1 / 2} z\right]} .
\end{gathered}
$$

We thus have explicit expressions for the evolution of the parameters of a Gaussian beam in a medium with independent linear and quadratic profiles of the gain and refractive index. Some consequences of these solutions are discussed in the following paragraphs.

The parameter $Q_{x}$ is considered first. Since in general both $k_{0}$ and $k_{2 x}$ may be complex, Eq. (23) represents a damped (or growing) nonsinusoidal oscillatory behavior, which may be characterized by a period $p_{x}$ and a damping length $l_{x}$ given by

$$
p_{x}=\pi\left[\operatorname{Re}\left(k_{2 x} / k_{0}\right)^{1 / 2}\right]^{-1}, l_{x}=\left[2 \operatorname{Im}\left(k_{2 x} / k_{0}\right)^{1 / 2}\right]^{-1}
$$

From Eqs. (21) and (23) one finds that the limiting value of the complex beam radius at large distances is

$$
1 / q_{x}(\infty)=\mp i\left(k_{2 x} / k_{0}\right)^{1 / 2}=1 / R_{x}(\infty)-i\left[\lambda_{m} /\left(\pi w_{x}^{2}(\infty)\right)\right]
$$

where the upper sign must be used if $\operatorname{Im}\left(k_{2 x} / k_{0}\right)^{1 / 2}$ $>0$ and the lower sign must be used if $\operatorname{Im}\left(k_{2 x} / k_{0}\right)^{1 / 2}$ $<0$. This steady-state solution can also be obtained directly from Eq. (15) by setting the derivative term equal to zero, but then one has no information about the choice of sign or the stability of the result. In a practical waveguiding problem it is also necessary that the steady-state solution represent a confined beam (real spot size). Equation (30) implies that this confinement condition is $\operatorname{Re} \pm\left(k_{2 x} / k_{0}\right)^{1 / 2}>0$, or with our sign convention ${ }^{6}$

$$
\operatorname{Im}\left(k_{2 x} / k_{0}\right)>0 .
$$

We now separate the propagation constant into its real and imaginary parts according to $k=\beta+i \alpha$. Then if the gain per wavelength is small $\left(\beta_{0} \gg \alpha_{0}\right)$, stability is assured by $\alpha_{2 x}>0$. Thus at distances large compared to $l_{x}$ the spot size and phase front curvature approach the values implied by Eq. (30) as long as $\alpha_{2 x}>0$. This result has been verified in high gain xenon lasers. ${ }^{5,6}$ In the limit of distances short compared to $l_{x}$ or when there is no gain profile $\left(l_{x} \rightarrow \infty\right)$ the beam parameter may oscillate without damping.

Figure 1 gives plots of the spot size in units of $2^{1 / 2}$ $\left(\beta_{0} \beta_{2 x}\right)^{-1 / 4}$ vs the normalized distance $z^{\prime}=\left(\beta_{2 x}\right)$ 


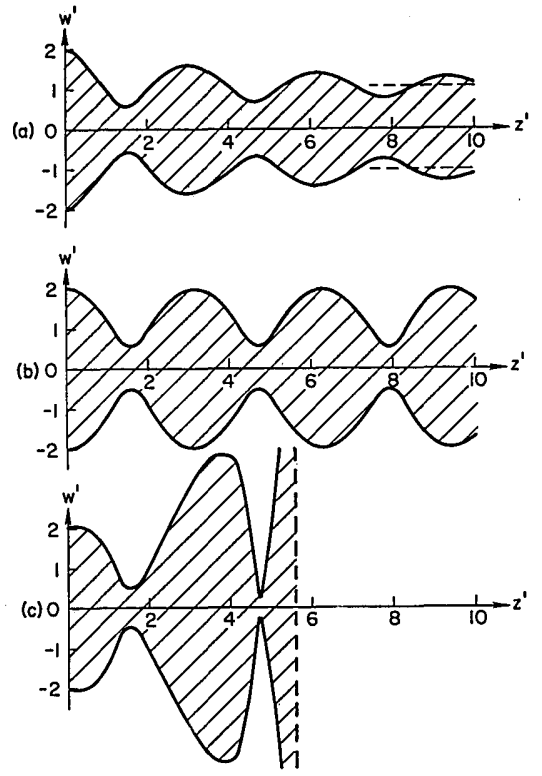

Fig. 1. Axial Gaussian beam in a complex lenslike medium showing the normalized spot size $w^{\prime}=2^{-1 / 2}\left(\beta_{0} \beta_{2 x}\right)^{1 / 4} w$ vs the normalized distance $z^{\prime}=\left(\beta_{2 x} / \beta_{0}\right)^{1 / 2} z$ for (a) $\alpha_{2 x} / \beta_{2 x}=0.1$, (b) $\alpha_{2 x} / \beta_{2 x}=0$, and (c) $\alpha_{2 x} / \beta_{2 x}=-0.1$. The dashed line in (c) marks the distance at which the spot size becomes infinite.

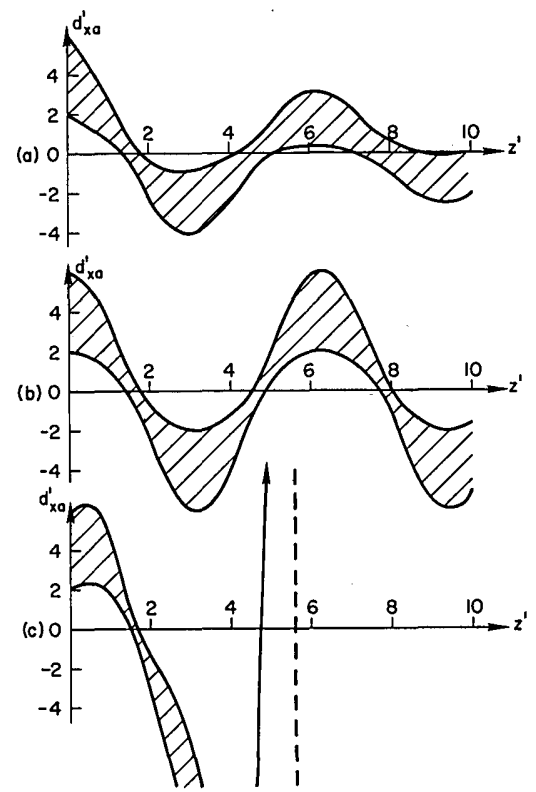

Fig. 2. Off-axis beam in a lenslike medium showing the normalized beam displacement in the $x$ direction vs $z^{\prime}$ for (a) $\alpha_{2 x} / \beta_{2 x}=$ 0.1 , (b) $\alpha_{2 x} / \beta_{2 x}=0$, and (c) $\alpha_{2 x} / \beta_{2 x}=-0.1$.

$\left.\beta_{0}\right)^{1 / 2} z$ for various values of $\alpha_{2 x} / \beta_{2 x}$. From these plots it is apparent that even when the gain profile is very weak compared to the index profile $\left(\alpha_{2 x} \ll \beta_{2 x}\right)$, still the gain can strongly affect the spot size of a propagating beam. Thus a gain profile in a waveguiding medium can serve to stabilize a beam as well as increasing its amplitude. To be useful in this function the medium must be longer than $l_{x}=[2 \mathrm{Im}$ - $\left.\left(k_{2 x} / k_{0}\right)^{1 / 2}\right]^{-1}$. If the gain profile is weak as in Fig. $1(\mathrm{a})$, the damping length is approximately $l_{x} \approx$ $\left(\beta_{2 x} / \beta_{0}\right)^{-1 / 2}\left(\beta_{2 x} / \alpha_{2 x}\right)$. On the other hand, it is also apparent that a negative gain profile $\left(\alpha_{2 x}<0\right)$ can rapidly lead to deterioration of the beam mode.

The behavior of the parameter $S_{x}$ is in many respects similar to that of $Q_{x}$, and $S_{x}$ also exhibits damped or growing nonsinusoidal oscillations. In the limit of large distances $\left(z \gg l_{x}\right)$ it follows from Eq. (27) that for a positive gain profile $\left(\alpha_{2 x}>0\right)$ in a focusing medium $\left(\beta_{2 x}>0\right) S_{x}$ approaches

$$
S_{x}(\infty)=-i\left[\left(k_{1 x} / 2\right)\left(k_{0} / k_{2 x}\right)^{1 / 2}\right] .
$$

The oscillation period is $2 p_{x}$, where $p_{x}$ is given in Eq. (29). Using Eq. (32) with Eq. (30) one finds that the displacement of the amplitude center of the beam $d_{x a}$ and the displacement of the center of curvature $d_{x p}$ approach the limits

$$
\begin{aligned}
d_{x a}(\infty) & =-\operatorname{Im}\left[\frac{-i k_{1 x}}{2}\left(\frac{k_{0}}{k_{2 x}}\right)^{1 / 2}\right] / \operatorname{Im}\left[-i\left(k_{0} k_{2 x}\right)^{1 / 2}\right] \\
& \approx-\frac{1}{2} \frac{\operatorname{Re}\left(k_{1 x} k_{2 x}{ }^{-1 / 2}\right)}{\operatorname{Re} k_{2 x}{ }^{1 / 2}}, \\
d_{x p}(\infty) & =-\operatorname{Re}\left[\frac{-i k_{1 x}}{2}\left(\frac{k_{0}}{k_{2 x}}\right)^{1 / 2}\right] / \operatorname{Re}\left[-i\left(k_{0} k_{2 x}\right)^{1 / 2}\right] \\
& \approx-\frac{1}{2} \frac{\operatorname{Im}\left(k_{1 x} k_{2 x}{ }^{-1 / 2}\right)}{\operatorname{Im} k_{2 x}{ }^{1 / 2}},
\end{aligned}
$$

where the approximation has been made that the gain per wavelength is small $\left(\beta_{0} \gg \alpha_{0}\right)$. In the opposite limit of distances short compared to $l_{x}$ or when $\alpha_{2 x}=0$, the parameters $d_{x a}(z)$ and $d_{x p}(z)$ may oscillate without damping.

Figure 2 gives plots of the beam displacement as a function of $z^{\prime}=\left(\beta_{2 x} / \beta_{0}\right)^{1 / 2} z$ for various values of $\alpha_{2 x} / \beta_{2 x}$ with $k_{1 x}=0$. Except for the initial displacement of four units the input conditions for these plots are the same as for those in Fig. 1. It is evident from the figure that even a very weak negative gain profile $\left(\left|\alpha_{2 x}\right| \ll \beta_{2 x}\right)$ may make the waveguide extremely unstable.

Figure 3 gives a plot of an off-axis beam in the limit that gain focusing is dominant $\left(\left|\alpha_{2 x}\right| \gg\left|\beta_{2 x}\right|\right)$. Evidently for a positive gain profile the beam displacement again rapidly decays to zero. These damped focusing effects are very important in high gain lasers. In such lasers the output properties should be relatively insensitive to mirror alignment, and this has been observed experimentally in our studies of xenon lasers at $3.51 \bar{\mu}$. These results should also be a useful for long-distance beam guiding, since the introduction of a medium with a gain or loss profile having $\alpha_{2 x}>0$ can compensate for any instabilities resulting from irregularities in the waveguiding medium. On the other hand, one can show that a gain profile tends to discriminate against the higher order Hermite-Gaussian or Laguerre-Gaussian modes, so any image information carried by the beam would be lost.

The results for the beam displacement simplify greatly in the limit that the propagation constant is 


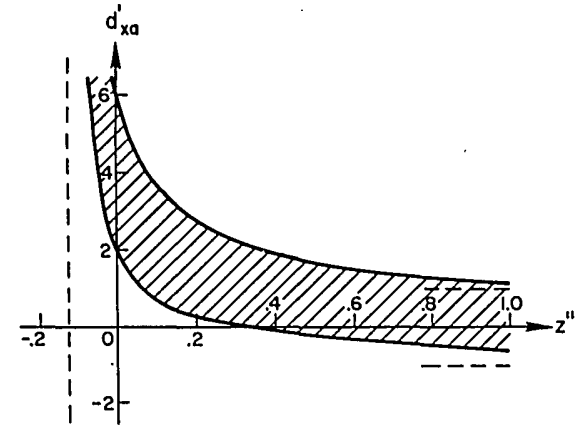

Fig. 3. Normalized beam displacement $d^{\prime}{ }_{x a}=$ $2^{-3 / 4}\left(\alpha_{2 x} \beta_{0}\right)^{1 / 4} d_{x a}$ and spot size vs $z^{\prime \prime}=\left(\alpha_{2 x} / 2 \beta_{0}\right)^{1 / 2} z$ for a gainfocused beam. Dashed lines are asymptotic limits. For $\alpha_{2 x}<0$ reverse direction of propagation.

real. This limit is important because most gas lenses and other waveguiding media are assumed to have a real index profile. Heuristic arguments suggest that for a medium of this type the center of a Gaussian beam should propagate along a trajectory that is a solution of the paraxial ray equation. ${ }^{12}$ Using the formalism developed here, this result can be rigorously derived. In particular it follows in a straightforward way from Eqs. (15) and (17) that for real $k$ the beam displacement $d_{x a}=-S_{x i} / Q_{x i}$ is governed by

$$
(d / d z) k_{0}\left(d d_{x a} / d z\right)=-\left(k_{1 x} / 2\right)-\left(k_{2 x} d_{x a}\right)
$$

In terms of the refractive index $n=n_{0}-1 / 2 n_{1 x} d_{x a}$ $1 / 2 n_{1 y} d_{y a}-1 / 2 n_{2 x} d_{x a}{ }^{2}-1 / 2 n_{2 y} d_{y a}{ }^{2}$ this result becomes

$$
\begin{aligned}
(! d / d z) n_{0}\left(d d_{x a_{1}} / d z\right)=\left(-n_{1 x} / 2\right) & -n_{2 x} d_{x a}=\left(\partial n / \partial d_{x a}\right) .
\end{aligned}
$$

The vector displacement $\bar{d}_{a}=\bar{i}_{x} d_{x a}+\bar{i}_{y} d_{y a}$ is governed by

$$
(d / d z) n_{0}\left(d^{\bar{d}} a / d z\right)=\bar{\nabla}_{\perp} n
$$

which is exactly the paraxial ray equation. Using the SELFOC fibers it has been verified experimentally that off-axis Gaussian beams in real lenslike media indeed propagate along ray trajectories. ${ }^{15}$ An important consequence of this result is that in optical systems containing real quadratic lenslike media the same $2 \times 2$ matrices that govern the evolution of the transverse beam properties according to the "ABCD law" also govern the raylike propagation of the beam center.

If the refractive index is independent of $z$, Eq. (36) reduces to

$$
\left(d^{2} / d z^{2}\right) d_{x a}+\left(n_{2 x} d_{x a} / n_{0}\right)=-n_{1 x} / 2 n_{0} .
$$

This is a linear second-order equation having the general solution

$$
\begin{aligned}
& d_{x a}(z)=\left[d_{x a}(0)+\left(n_{1 x} / 2 n_{2 x}\right)\right] \cos \left[\left(n_{2 x} / n_{0}\right)^{1 / 2} z\right] \\
& \quad+d_{x a}{ }^{\prime}(0)\left(n_{0} / n_{2 x}\right)^{1 / 2} \sin \left[\left(n_{2 x} / n_{0}\right)^{1 / 2} z\right]-\left(n_{1 x} / 2 n_{2 x}\right),
\end{aligned}
$$

where $d_{x a}(0)$ and $d_{x a}(0)$ are, respectively, the initial beam displacement and slope. Thus, the beam center oscillates sinusoidally about the limiting displacement $d_{x a}(\infty)=-n_{1 x} / 2 n_{2 x}$ of Eq. (33) independent of the beam dimensions. If $n_{1 x}=0$, this result simplifies to

$$
\begin{aligned}
d_{x a}(z)=d_{x a}(0) & \cos \left[\left(n_{2 x} / n_{0}\right)^{1 / 2} z\right] \\
& +d_{x a}{ }^{\prime}(0)\left(n_{0} / n_{2 x}\right)^{1 / 2} \sin \left[\left(n_{2 x} / n_{0}\right)^{1 / 2} z\right] .
\end{aligned}
$$

In a defocusing lenslike medium with $n_{2 x}<0$ similar expressions are obtained in terms of hyperbolic sine and cosine functions. If instead $n_{2 x}=0$, the trajectory is the parabola

$$
d_{x a}=d_{x a}(0)+d_{x a}{ }^{\prime}(0) z-\left[n_{1 x} /\left(4 n_{0}\right)\right] z^{2} .
$$

The behavior of the parameter $P$ is similar to that of $Q_{x}$ and $S_{x}$. The phase too exhibits a damped oscillatory behavior. It follows from Eq. (28) that for distances that are large compared to $l_{x}$ and $l_{y}$ the phase develops according to

$$
\begin{aligned}
P(z)=\left(k_{1 x}{ }^{2} z / 8 k_{2 x}\right) & +\left(k_{1 y}{ }^{2} z / 8 k_{2 y}\right) \\
& +\frac{1}{2}\left(k_{2 x} / k_{0}\right)^{1 / 2} z+\frac{1}{2}\left(k_{2 y} / k_{0}\right)^{1 / 2} z .
\end{aligned}
$$

Thus the presence of linear or quadratic variations of the propagation constant may affect both the phase velocity and the gain of a steady-state confined beam. For distances that are short compared to $l_{x}$ and $l_{y}$ or when $\alpha_{2 x}=\alpha_{2 y}=0$, the complex phase of the beam oscillates without damping, and the detailed behavior follows from Eq. (28).

In summary, we have described in this section general solutions of the equations governing the parameters $Q, S$, and $P$ of a Gaussian beam in a lenslike medium having linear and quadratic variations of the complex propagation constant. It was shown that all the properties of the beam including the size, location, and phase damp toward a steady-state behavior if $\alpha_{2 x}, \alpha_{2 y}>0$. In the special case that there is no gain or loss profile, the beam oscillates without damping and the displacement of the beam is governed by the paraxial ray equation. If $\alpha_{2 x}, \alpha_{2 y}<0$, the propagation is extremely unstable.

\section{Axial Beams in Focusing Media}

In the preceding discussion it has been assumed that the center of the Gaussian beam is in general not coincident with the $z$ axis. Here we restrict attention to axial beams in quadratic lenslike media $\left(k_{1 x}=k_{1 y}=0\right)$. With these restrictions the mathematics simplifies and it becomes straightforward to treat higher order modes. An example is also given of the calculation of the weak $z$ components of the fields.

We look first for the cylindrical field components of a set of beam modes. The starting point is the general wave equation given in Eq. (2). If the $\bar{\nabla} \times$ $\bar{\nabla} \times$ operator is expanded in cylindrical coordinates, one finds that this wave equation can be written 


$$
\begin{aligned}
\nabla_{c}^{2} \bar{E}+k^{2} \bar{E}= & 1 / r^{2}\left\{\bar{i}_{r}\left[E_{r}+2\left(\partial E_{\phi} / \partial \phi\right)\right]\right. \\
& \left.+\bar{i}_{\phi}\left[E_{\phi}-2\left(\partial E_{r} / \partial \phi\right)\right]\right\} \\
- & \bar{\nabla}[(\bar{\nabla} \epsilon / \epsilon) \cdot \bar{E}]-(\bar{\nabla} \mu / \mu) \times \bar{\nabla} \times \bar{E},
\end{aligned}
$$

where we are defining $\nabla_{c}^{2}$ as the Laplacian operator in cylindrical coordinates operating on each of the cylindrical components of $\bar{E}$. This equation differs from Eq. (3) for the Cartesian field components in the coupling term on the right-hand side involving $E_{r}$ and $E_{\phi}$.

As indicated previously, the gradient terms in the wave equations for the transverse field components may be neglected if the changes in $\epsilon$ and $\mu$ per wavelength are small. Accordingly, we obtain from Eq. (43) the two coupled equations for $E_{r}$ and $E_{\phi}$

$$
\begin{aligned}
& \nabla_{c}^{2} E_{r}+k^{2} E_{r}=1 / r^{2}\left[E_{r}+2\left(\partial E_{\phi} / \partial \phi\right)\right], \\
& \nabla_{c}^{2} E_{\phi}+k^{2} E_{\phi}=1 / r^{2}\left[E_{\phi}-2\left(\partial E_{r} / \partial \phi\right)\right] .
\end{aligned}
$$

These equations are solved if the field components are related to a scalar function $T_{\mp}$ by the relations

$$
\begin{aligned}
& E_{r}=\mp i T_{\mp}(r, z) \exp (-i n \phi), \\
& E_{\phi}=-T_{\mp}(r, z) \exp (-i n \phi),
\end{aligned}
$$

where $T_{\mp}$ is any solution of

$$
\begin{aligned}
\left(\partial^{2} T_{\mp} / \partial r^{2}\right)+(1 / r)\left(\partial T_{\mp} / \partial r\right)-\left[(n \mp 1)^{2} / r^{2}\right] T T_{\mp} \\
+\left(\partial^{2} T_{\mp} / \partial z^{2}\right)+k^{2} T_{\mp}=0 .
\end{aligned}
$$

These modes have helical phase fronts and are either left-hand or right-hand polarized depending on whether the upper or lower signs are chosen in Eqs. (46) and (47). Alternatively one could choose $E_{r}=$ $\mp i T_{\mp}^{*} \exp (i n \phi), E_{\phi}=T_{\mp} \exp (i n \phi)$ or for nonrotating polarization $E_{r}=\mp T_{\mp} \sin n \phi, E_{\phi}=-T_{\mp} \cos n \phi$. For all of these descriptions of the fields the scalar function $T_{\mp}$ is governed by Eq. (47).

Besides the choice of polarization, Eq. (47) also admits of complex $z$-dependent $k$ and is thus the cylindrical-component analog of Eq. (4), the Cartesian-component scalar wave equation. An indirectly derived special case of this result is given as Eq. (33) of Ref. 16. General solutions of Eq. (47) may be obtained as in Sec. II. For quadratic lenslike media governed by $k^{2}(r, z)=k_{0}(z)\left[k_{0}(z)-k_{2}(z) r^{2}\right]$ the substitution

$$
T_{\mp}=U_{\mp}(r, z) \exp \left[-i \int k_{0}(z) d z\right]
$$

reduces Eq. (47) to

$$
\begin{aligned}
& \left(\partial^{2} U_{\mp} / \partial r^{2}\right)+(1 / r)\left(\partial U_{\mp} / \partial r\right)-\left[(n \mp 1)^{2} / r^{2}\right] U_{\mp} \\
& -i\left(d k_{0} / d z\right) U_{\mp}-2 i k_{0}\left(\partial U_{\mp} / \partial z\right)-k_{0} k_{2} r^{2} U_{\mp}=0,
\end{aligned}
$$

where $U_{\mp}$ has been assumed to vary so slowly with $z$ that its second derivative can be neglected. The steady-state beam modes in a medium with constant real $k$ are considered here. For this case one finds by substitution that the solutions of Eq. (49) are

$$
\begin{array}{r}
U_{\mp}=\left(2 r^{2} / w^{2}\right)^{(n \mp 1) / 2} L_{m}{ }^{n \mp 1}\left(2 r^{2} / w^{2}\right) \exp \left(-r^{2} / w^{2}\right) \\
\times \exp \left[i(2 m+1+n \mp 1)\left(k_{2} / k_{0}\right)^{1 / 2} z\right],
\end{array}
$$

where $w=2^{1 / 2}\left(k_{0} k_{2}\right)^{-1 / 4}$ is the steady-state Gaussian spot size of Eq. (30) and $L_{m}{ }^{n \mp 1}$ are the general- ized Laguerre polynomials. The integer $m$ may take on any value $m=0,1,2, \ldots$ and for $n=0$ the lower sign must be chosen.

As indicated in Sec. II the remaining field components can be found from the transverse components of the electric field by means of Maxwell's equations. If Eqs. (1) are applied to Eqs. (46) and (48), one finds that the transverse components of the magnetic field are given approximately by

$$
\begin{aligned}
& H_{r}=(\epsilon / \mu)^{1 / 2} T_{\mp} \exp (-i n \phi), \\
& H_{\phi}=\mp i(\epsilon / \mu)^{1 / 2} T_{\mp} \exp (-i n \phi) .
\end{aligned}
$$

and the longitudinal fields are

$$
\begin{gathered}
E_{z}=\mp 1 / k_{0}\left\{\left(\partial T_{\mp} / \partial r\right)+[(1 \mp n) / r] T_{\mp}\right\} \exp (-i n \phi), \\
H_{z}=-i / \omega \mu\left\{\left(\partial T_{\mp} / \partial r\right)+[(1 \mp n) / r] T_{\mp}\right\} \exp (-i n \phi) .
\end{gathered}
$$

Using Eq. (50) and the recursion relations for the Laguerre polynomials it follows that the factor appearing in Eqs. (52) has the value

$$
\begin{aligned}
& \left(\partial T_{\mp} / \partial r\right)+[(1 \mp n) / r] T_{\mp}=1 / r\{[2 m+(n+1) \\
& \left.\times(1 \mp 1)-\left(2 r^{2} / w^{2}\right)\right] L_{m}{ }^{n \mp 1}\left(2 r^{2} / w^{2}\right)-2(m+n \mp 1) \\
& \left.\quad \times L_{m-1}{ }^{n \mp 1}\left(2 r^{2} / w^{2}\right)\right\} \cdot\left(2 r^{2} / w^{2}\right)^{(n \mp 1) / 2} \exp \left(-r^{2} / w^{2}\right) \\
& \quad \times \exp \left(-i k_{0} z\right) \exp \left[i(2 m+1+n \mp 1)\left(k_{2} / k_{0}\right)^{1 / 2} z\right] .
\end{aligned}
$$

When $m=0$, the polynomial $L_{m-1}{ }^{n \mp 1}$ must be set equal to zero in this result. This form for the $z$ components of the fields can also be obtained directly from the $z$ component of Eq. (43) using Eqs. (1) provided that the gradient terms are not neglected. As indicated previously these coupling terms are not negligible when considering the weaker longitudinal field components.

From a comparison of Eqs. (46) and (52) one finds that for low order modes the longitudinal fields are smaller than the transverse fields by a factor of roughly $k_{0} r \approx k_{0} w=\left(2 k_{0}\right)^{1 / 2}\left(k_{0} / k_{2}\right)^{1 / 4}$. In typical applications involving optical lasers $k_{0} \approx 10^{7} \mathrm{~m}^{-1}$ and $w \approx 10^{-3} \mathrm{~m}$, so that the longitudinal fields are smaller by a factor of $10^{4}$. For such situations the waveguided beams may for all practical purposes be regarded as TEM modes. It should be noted that the presence of longitudinal field components is not a characteristic feature of waveguided beams. It is clear from the derivation that such components will be present with all beams of finite transverse extent, even if they are propagating in free space.

Similar derivations can be readily performed to obtain the Cartesian field components of a set of higher order axial beam modes. The analysis is simpler than for the previous case, and the $x$ and $y$ field components are governed by Eq. (4). The Laplacian operator may be conveniently expressed in Cartesian or cylindrical coordinates and the remaining field components may be found from Eqs. (1). The Hermite-Gaussian and Laguerre-Gaussian solutions of the scalar wave equation for this case are known. ${ }^{17}$ We conclude this section by observing that the wave equation is the appropriate starting point for all problems involving the propagation of light 
beams in media with slow spatial variations of the propagation constant, and the techniques outlined here apply in principle even if the beam parameters and medium properties are not constant.

\section{Thin-Medium Approximation}

An important application of the formalism governing the interaction of an inhomogeneous medium and a Gaussian light beam is the probing of a medium with a laser beam. We consider here the situation in which the beam diameter is small compared to the scale of the inhomogeneities so that Eqs. (15)-(19) will apply. It is also assumed that the over-all dimensions of the medium are so small that the parameters $Q, S$, and $P$ of the beam change only slightly as the beam propagates through the medium. The results will be applicable, for example, to plasma diagnosis where the strength of the lightplasma interaction can be controlled by proper choice of the laser frequency relative to the plasma frequency. It has been shown experimentally that significant deflection of a light beam is readily obtained in pulsed ${ }^{18}$ and $\mathrm{cw}^{19}$ plasmas. The examples considered here concern first the focusing and then the deflection of a beam caused by an axially symmetric plasma.

If $k_{0}$ is nearly constant in the inhomogeneous medium, Eq. (15) can be written

$$
(d / d z)\left(1 / q_{x}\right)=-\left(1 / q_{x}\right)^{2}-\left(k_{2 x} / k_{0}\right) .
$$

Therefore, the change in the beam parameter in passing through the medium (besides that due to free space diffraction) is

$$
\Delta(1 / q)=-\int\left(k_{2 x} / k_{0}\right) d z .
$$

where the integration extends across the medium. Thus, the medium acts like a thin lens of complex focal length $\left[\int\left(k_{2 x} / k_{0}\right) d z\right]^{-1}$.

We assume now that the medium has a cylindrical geometry as shown schematically in Fig. 4. This configuration would apply to arc plasmas under a variety of conditions. Comparing the wavenumber in the vicinity of the beam $k=k_{0}-1 / 2 k_{1 x}\left(x-x_{0}\right)-$ $1 / 2 k_{2 x}\left(x-x_{0}\right)^{2}$ with a Taylor expansion, Eq. (55) can be written

$$
\Delta\left(\frac{1}{q}\right)=\left.\frac{1}{k_{0}} \int_{-\infty}^{\infty} \frac{\partial^{2} k(x, z)}{\partial x^{2}}\right|_{x_{0}} d z
$$

where $x_{0}$ is the distance of the beam from the axis of the cylindrical medium. Since the plasma has cylindrical symmetry, the complex wavenumber can be expressed as a function of the coordinate $r=\left(x^{2}\right.$ $\left.+z^{2}\right)^{1 / 2}$. Thus, Eq. (56) becomes

$$
\begin{aligned}
& \Delta\left[\frac{1}{q\left(x_{0}\right)}\right]=\frac{1}{k_{0}} \int_{-\infty}^{\infty}\left[\frac{x^{2} \partial^{2} k}{r^{2} \partial r^{2}}+\left(\frac{1}{r}-\frac{x^{2}}{r^{3}}\right) \frac{\partial k}{\partial r}\right]_{x_{0}} d z \\
& =\frac{2}{k_{0}} \int_{x_{0}}^{\infty}\left\{\left[\frac{x_{0}^{2}}{r} \frac{d^{2} k}{d r^{2}}+\left(1-\frac{x_{0}^{2}}{r^{2}}\right) \frac{d k}{d r}\right] /\left(r^{2}-x_{0}^{2}\right)^{1 / 2}\right\} d r .
\end{aligned}
$$

If the plasma properties were known, this result would yield immediately the transmission characteristics of a Gaussian laser beam. On the other hand,
Eq. (57) could in principle be inverted so that the unknown function $k(r)$ could be obtained from the experimentally determined function $\Delta\left[q\left(x_{0}\right)\right]^{-1}$. Thus we have a procedure by means of which $k(r)$ for a cylindrical medium can be determined from measurements of the focusing or defocusing of a Gaussian beam. In practice a numerical analysis would be required. For a plasma a knowledge of the complex wavenumber yields information about the electron density and temperature.

It turns out that information about the properties of a localized medium can be obtained in a more straightforward way from a consideration of the beam deflection. We consider here only the case of a lossless medium, so that the beam displacement is governed by Eq. (38). Using the previous approximation that the beam is displaced negligibly in crossing the medium $\left(d_{x a} \approx 0\right)$, Eq. (38) reduces to

$$
d \theta\left(x_{0}, z\right) / d z=-\left(n_{1 x} / 2 n_{0}\right)
$$

where the angle $\theta=d d_{x a} / d z$ is defined in Fig. 4 . Integrating across the medium yields the total deflection angle

$$
\theta\left(x_{0}\right)=-\frac{1}{2 n_{0}} \int_{-\infty}^{\infty} n_{1 x} d z=\left.\frac{1}{n_{0}} \int_{-\infty}^{\infty} \frac{\partial n}{\partial x}\right|_{x_{0}} d z .
$$

The deflection angle can be determined experimentally and is assumed here to be known. Using the cylindrical symmetry Eq. (59) can be written

$$
\theta\left(x_{0}\right)=\frac{x_{0}}{n_{0}} \int_{-\infty}^{\infty} \frac{1}{r} \frac{d n}{d r} d z=\frac{2 x_{0}}{n_{0}} \int_{x_{0}}^{\infty} \frac{(d n / d r) d r}{\left(r^{2}-x_{0}^{2}\right)^{1 / 2}} .
$$

This result is much simpler than Eq. (57). In fact Eq. (60) can be put in the form of Abel's integral equation, and thus it can be inverted explicitly. The result is ${ }^{20}$

$$
n(r)=-\frac{n_{0}}{\pi} \int_{r}^{\infty} \frac{\theta\left(x_{0}\right) d x_{0}}{\left(x_{0}^{2}-r^{2}\right)^{1 / 2}}
$$

Hence, the radial profile of the refractive index may be readily determined by measuring the deflection of a laser beam as it is scanned across a plasma and substituting the results in Eq. (61). This integration may be performed analytically for a wide range of functions $\theta\left(x_{0}\right)$, and in any case numerical integra-

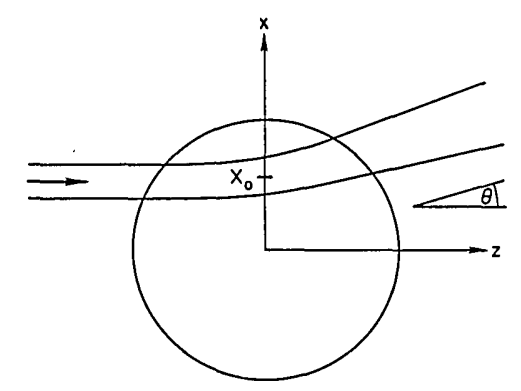

Fig. 4. Schematic drawing of a typical experiment involving the interaction of a Gaussian laser beam and a cylindrical plasma. 
tion is always possible. In a previous ray treatment of the scattering of light from cylindrical plasmas with real refractive index it was assumed that the rays could be deflected through large angles. ${ }^{21}$ With that analysis an explicit formula for the refractive index (electron density) could not be obtained. In practical plasma situations using lasers deflection angles are small except with far-infrared and submillimeter radiation, ${ }^{22}$ so the present formalism almost always applies.

In summary, the methods of this section may be applied in any situation involving the propagation of Gaussian light beams through localized inhomogeneous media having slow variations of the loss or refractive index. The examples discussed have dealt with the important problem of diagnosis of cylindrical plasmas.

\section{Conclusion}

In this work we have developed a general formalism for treating the propagation of Gaussian beams of light in complex inhomogeneous media. The results are useful because the output of most lasers is in the form of Gaussian beams and in many practical types of media such beams remain Gaussian in spite of changes of amplitude, direction, and phase. A set of first-order ordinary differential equations have been derived that govern all the properties of these beams. The equations have been solved exactly for the propagation of general off-axis modes in lenslike media, and the same methods are applicable to higher order Laguerre-Gaussian and Hermite-Gaussian beams. The results also lead to a straightforward procedure for the diagnosis of localized inhomogeneous media using laser beams. The problems that have been discussed concerning stable and unstable lenslike waveguides and cylindrical plasmas are important representative examples of the usefulness of these methods.

The author wishes to acknowledge valuable discussions with D. L. Jassby.
This work was supported in part by the National Science Foundation.

\section{References}

1. G. D. Boyd and J. P. Gordon, Bell Sys. Tech. J. 40, 489 (1961).

2. G. Goubau and F. Schwering, IRE Trans. Antennas Propag. AP-9, 248 (1961).

3. B. W. McCaul, Appl. Opt. 9, 653 (1970).

4. H. Kogelnik, Appl. Opt. 4, 1562 (1965).

5. L. W. Casperson and A. Yariv, Appl. Phys. Lett. 12, 355 (1968).

6. L. W. Casperson and A. Yariv, Appl. Opt. 11, 462 (1972).

7. L. A. Schlie and J. T. Verdeyen, IEEE J. Quantum Electron. QE-5, 21 (1969).

8. L. M. Osterink and J. D. Foster, Appl. Phys. Lett. 12, 128 (1968).

9. D. W. Berreman, J. Opt. Soc. Am. 55, 239 (1965).

10. D. Marcuse and S. E. Miller, Bell Syst. Tech. J. 43, 1759 (1964).

11. E. A. J. Marcatili, Bell Syst. Tech. J. 43, 2887 (1964).

12. P. K. Tien, J. P. Gordon, and J. R. Whinnery, Proc. IEEE 53, 129 (1965).

13. T. Uchida, M. Furukawa, J. Kitano, K. Koizumi, and H. Matsumura, paper presented at the IEEE/Optical Society of America Joint Conference on Laser Engineering Applications, May 1969, Washington, D.C.

14. A. Sommerfeld and J. Runge, Ann. Phys. 35, 290 (1911).

15. A. N. Rosen, Appl. Opt. 11, 946 (1972).

16. C. N. Kurtz and W. Streifer, IEEE Trans. Microwave Theory Tech. MTT-17, 11 (1969).

17. H. Kogelnik and T. Li, Appl. Opt. 5, 1550 (1966).

18. G. Lampis and S. C. Brown, Phys. Fluids 11, 1137 (1968).

19. M. Nakatsuka, M. Yokoyama, Y. Izawa, K. Toyoda, and C. Yamanaka, Phys. Lett. 37A, 169 (1971).

20. A related transform is given by K. Bockasten, J. Opt. Soc. Am. 51, 943 (1961).

21. J. Shmoys, J. Appl. Phys. 32, 689 (1961). Equation (21) $\operatorname{simplifies}$ to $v(w)=\exp$

$$
\exp \left[-\frac{w^{1 / 2}}{\pi} \int_{0}^{w} \frac{\theta(x) d x}{x(w-x)^{1 / 2}}\right]
$$

22. D. L. Jassby and M. E. Marhic, J. Appl. Phys. 43, 4586 (1972).

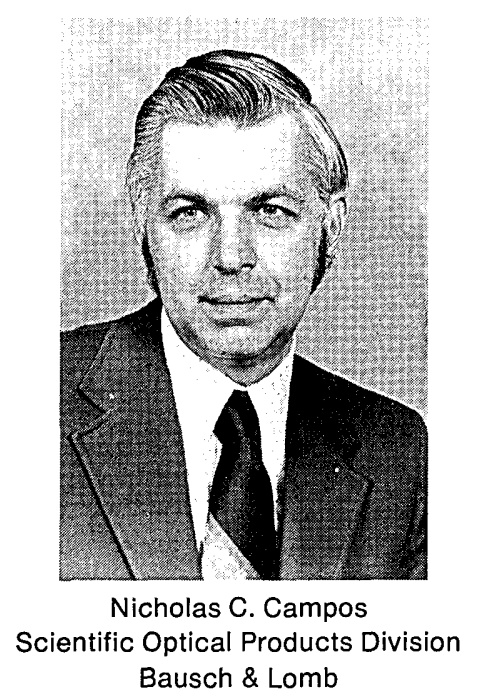

